Reinhold F. Glei

\title{
Tertius motus: Die Erklärung der Präzession im Anti-Lucretius des Melchior de Polignac
}

Bei der Darstellung naturwissenschaftlicher Sachverhalte im Lehrgedicht ${ }^{1}$ sind grundsätzlich zwei Ansätze zu unterscheiden: der phänomenologische und der kausale. Zunächst ist zu fragen, ob der zu erklärende Sachverhalt korrekt (das heißt nach Maßgabe der zeitgenössischen Fachwissenschaft) erfasst und referiert ist; gewisse poetische Lizenzen sind dabei zu konzedieren, aber das Maß der Genauigkeit kann gleichzeitig als Maß für die poetische Qualität des Lehrgedichts dienen, denn für die Poetologie des (antiken wie neuzeitlichen) Lehrgedichts muss prinzipiell von einem Ideal wissenschaftlicher Korrektheit ausgegangen werden. Die Glaubwürdigkeit des Lehrgestus hängt entscheidend von der Verlässlichkeit der Sachinformation ab, auch wenn diese gar nicht das eigentliche didaktische Objekt sein sollte (wie es etwa beim sogenannten transparenten Typ der Fall ist). ${ }^{2}$ Hesiod und Vergil wollten gewiss nicht die Bauern über die Landwirtschaft belehren, wie schon Seneca feststellte, ${ }^{3}$ aber wenn sie agrarisches Wissen nicht korrekt reproduziert hätten, wäre ihre moralische Botschaft nicht ernstgenommen worden. Arat wurde bekanntlich dafür kritisiert, dass er astronomische Fehler begangen und die Phänomene eben nicht vollständig korrekt beschrieben habe; ${ }^{4}$ die alexandrinische l'art pour l'art-Hypothese (Arat habe gerade als Nichtfachmann eine ihm fremde Disziplin ,lehren' wollen) trägt deutlich apologetische Züge, ${ }^{5}$ und die lateinischen

1 Zu grundsätzlichen Fragen vgl. den Artikel von Martin Korenjak: Explaining Natural Science in Hexameters. Scientific Didactic Epic in the Early Modern Era. In: Humanistica Lovaniensia 68.1 (2019), S. 135-175.

2 Vgl. Bernd Effe: Dichtung und Lehre. Untersuchungen zur Typologie des antiken Lehrgedichts. München 1977.

3 Sen. Ep. mor., 86,15: „Vergilius [...], qui non quid verissime sed quid decentissime diceretur aspexit, nec agricolas docere voluit sed legentes delectare.“

4 Vgl. Cic. De or., 1,69: „constat inter doctos, hominem ignarum astrologiae ornatissimis atque optimis versibus Aratum de caelo stellisque dixisse.“

5 Die anekdotische Überlieferung findet sich in den antiken Viten, die den Ausgaben der Phainomena vorangestellt waren, siehe Georg Knaack: Aratos (6). In: RE 3 (1895), S. 391-399. 
Arat-Bearbeitungen zeigen die Tendenz, inhaltliche Fehler des Originals zu verbessern. ${ }^{6}$

Der kausale Ansatz steht auf einem völlig anderen Blatt. Viele Lehrgedichte beschränken sich dezidiert auf das Phänomenologische und nehmen kausale Erklärungen gar nicht in den Blick; dies ist bei landwirtschaftlichem oder generell bei praktischem Alltagswissen durchaus verständlich und naheliegend, da die komplexen Vorgänge hinter den Phänomenen oft undurchschaubar und für den Durchschnittsleser auch nicht relevant sind. Auch in den astronomischen Lehrgedichten kommt es eher auf praktische Himmelsbeobachtung als auf wissenschaftliche Erklärungen an - für den Seemann ist es unerheblich, ob sich die Sonne um die Erde dreht oder umgekehrt. Anders sieht es jedoch bei denjenigen Lehrgedichten aus, die eine bestimmte philosophische Lehre vertreten bzw. verbreiten wollen: Sie nehmen die Phänomene nur als Ausgangspunkt, um ihre Weltsicht zu entwickeln. Zu diesem Zweck müssen sie sich der Begründung der Phänomene widmen, d. h. den Leser in einem rationalen Diskurs von der Wahrheit ihrer Lehre überzeugen. Mit anderen Worten: Sie stellen Hypothesen auf, die mithilfe der Phänomene bzw. phänomenologischer Betrachtungen bewiesen werden sollen; umgekehrt müssen sich aber auch die Hypothesen den Phänomenen ,stellen', d. h. sie könnten schlimmstenfalls durch die Beobachtung auch falsifiziert werden.

Das Lehrgedicht des Lukrez ist ohne Zweifel das herausragende Beispiel für eine einheitliche kausale Erklärung nahezu sämtlicher Naturphänomene durch die demokritisch-epikureische Atomlehre. Die Naturerkenntnis ist dabei freilich kein Selbstzweck, sondern, Hand in Hand mit der Poetisierung, ein Mittel zur Plausibilisierung ethischer, anthropologischer und (anti-)theologischer Positionen. ${ }^{7}$ Dieselbe Intention, nur mit umgekehrtem Vorzeichen, verfolgt dementsprechend der Anti-Lucretius des Kardinals Melchior de Polignac (1661-1741): Mithilfe rationaler Deutung von Naturphänomenen (auf der Grundlage zeitgenössischer wissenschaftlicher Erkenntnisse) versucht der Autor, eine anti-lukrezische bzw. anti-epikureische Ethik, Anthropologie und Theologie zu begründen (so programmatisch im ersten Buch, De voluptate betitelt). ${ }^{8}$ Dabei verselbstständigen

6 Das ist schon für Ciceros Übersetzung der Fall, spätere Arat-Bearbeitungen gehen noch weiter. Der Astronom Hipparch verfasste im zweiten Jahrhundert v.Chr. einen kritischen Kommentar zu Arat, in dem er ihm seine Fehler nachwies.

7 Siehe dazu grundlegend: Hans Blumenberg: Schiffbruch mit Zuschauer. Paradigma einer Daseinsmetapher. Frankfurt a.M. 1979; außerdem Verf.: Erkenntnis als Aphrodisiakum. Poetische und philosophische voluptas bei Lukrez. In: Antike \& Abendland 38 (1992), S. 82-94.

8 Dabei reagiert der Autor insbesondere auf die ,Rehabilitierung Epikurs und seiner Ethik durch Pierre Gassendi (1592-1655); vgl. den Überblick von Olivier Bloch: Pierre Gassendi. 
sich allerdings oft die naturwissenschaftlichen Erörterungen so weit, dass der Zusammenhang mit der religiösen Grundintention völlig aus dem Blick gerät: Man gewinnt den Eindruck, dass sich der Kardinal meist von der Wissenschaft um ihrer selbst willen begeistern lässt, wie man es für das Zeitalter der wissenschaftlichen Revolution als typisch anzusehen geneigt ist. In den Büchern 2-8 treten daher die moralischen und theologischen Aspekte zugunsten der Naturwissenschaft, die sowohl in den großen Themen als auch in zahlreichen Detailfragen präsent ist, ganz zurück. ${ }^{9}$ Wie sein erklärter Feind, der antike Dichter Lukrez, achtet der Kardinal dabei stets auf zweierlei: auf strikte Korrektheit nach Maßgabe des zeitgenössischen Wissensstandes und auf ebenso strikte rationale Plausibilität.

Entscheidend für die Beurteilung der sachlichen ,Korrektheit‘ ist die chronologische Verortung: Das Lehrgedicht erschien zwar erst postum 1747, verfasst wurde es aber bereits rund 50 Jahre früher, nämlich nach einer eindrücklichen Begegnung des Autors mit Pierre Bayle 1697 in den Niederlanden, als Polignac, wegen eines diplomatischen Misserfolgs bei Ludwig XIV. in Ungnade gefallen, fünf Jahre in der Abtei Bonport in der Normandie verbringen musste. ${ }^{10}$ Das Lehrgedicht ist vermutlich damals im Wesentlichen fertiggestellt worden, denn Spuren späterer Bearbeitung lassen sich kaum finden. Das neunte Buch blieb unvollendet (nur das Proömium ist erhalten), ${ }^{11}$ und manche Dubletten, Brüche und Widersprüche zeugen vom Zustand der Unfertigkeit - auch darin ist Polignac ironischerweise seinem, Vorbild‘ Lukrez ähnlich.

In: Die Philosophie des 17. Jahrhunderts. 2: Frankreich und Niederlande. Erster Halbband. Hg. von Jean-Pierre Schobinger. Basel 1993, S. 201-230.

9 Ein Beispiel ist die Diskussion um Urzeugung und Fortpflanzung und die Kontroverse zwischen ,Ovulisten' und ,Spermatisten“ im 7. Buch; siehe dazu Verf.: Novus orbis: Melchior de Polignac über das Mikroskop. In: Acta Conventus Neo-Latini Abulensis. Proceedings of the Tenth International Congress of Neo-Latin Studies, Avila 4-9 August 1997. Hg. von Rhoda Schnur et al. Tempe, Arizona 2000, S. 283-291.

10 Zur Editionsgeschichte, die noch nicht vollständig aufgearbeitet ist, vgl. meinen in Anm. 9 genannten Aufsatz sowie Claudia Schindler: Aeternitatis et immortalitatis desiderio ardere homines. Unsterblichkeitsbeweise in der neulateinischen Lehrdichtung von Paleario bis Polignac. In: Wolfenbütteler Renaissance-Mitteilungen 35.2 (2014), S. 125-153, insbes. S. 145-146, mit der älteren Literatur.

11 AL 9,1-24a. Danach bricht der Text ab. Die folgende, anstelle des 9. Buches gesetzte Conclusio totius operis stammt vermutlich nicht von Polignac selbst, sondern ist eine Kompilation des postumen Herausgebers, des Abbé de Rothelin. Es wurde sogar die These vertreten, die Bücher 6-9 stammten insgesamt von Rothelin: vgl. Wolfgang Bernard Fleischmann: Zum Anti-Lucretius des Kardinals de Polignac. In: Romanische Forschungen 77 (1965), S. 42-63. Nähere Untersuchungen dazu fehlen. 
Die Spannung zwischen dem phänomenologischen und dem kausalen Ansatz lässt sich gut an der Diskussion um die Newton'sche Gravitationslehre erkennen: Polignac bestreitet nicht, dass sich damit die Phänomene korrekt beschreiben und insbesondere berechnen lassen, aber er kritisiert immer wieder die in seinen Augen irrationale Annahme einer unerklärlichen Fernwirkung durch den (angeblich) leeren Raum. ${ }^{12}$ Tatsächlich hat Newton dafür keine Begründung gegeben (vgl. seinen berühmten Ausspruch hypotheses non fingo), was Polignac als den entscheidenden Mangel dieser Theorie ansieht, den sie im Übrigen mit der lukrezischen bzw. epikureischen Kinetik teile. ${ }^{13}$ Demgegenüber vertritt (und erläutert) Polignac die zwar kompliziertere, aber für ihn kausal eher nachvollziehbare Cartesianische Wirbeltheorie (AL 4,575-932). ${ }^{14}$ Man liest immer wieder, Polignac hänge hiermit starrsinnig einer inzwischen obsoleten, durch Newton überholten Lehre an, ${ }^{15}$ aber das trifft keineswegs zu: Zum einen wurden beide Theorien bis weit ins achtzehnte Jahrhundert hinein noch als prinzipiell gleichwertig diskutiert; ${ }^{16}$ erst aus heutiger Sicht erscheint die Wirbeltheorie als antiquiert und eher naiv. Zum anderen gab es tatsächlich vor Einstein keine rationale Erklärung für die Gravitationstheorie. Erst durch die Allgemeine Relativitätstheorie (zuerst 1915) wurde eine solche möglich, und es sollte noch weitere 100 Jahre dauern, bis sie durch das LIGO-Team experimentell bewiesen wurde. ${ }^{17}$ Zum Zeitpunkt der Publikation des Anti-Lucretius (1747) setzten zwar nur noch wenige Gelehrte gegen Newton auf Descartes, aber es besteht kein Grund, über Polignacs angeblich anachronistische Verteidigung der Wirbeltheorie, die schon fast 50 Jahre früher entstand, die Nase zu rümpfen. Im

12 Vgl. dazu insbesondere den in das 2. Buch eingefügten Exkurs über Newtons Theorie des Vakuums und der Gravitation (AL 2,865-991), die Polignac als „Märchen“ (fabula) bezeichnet. 13 Darüber handelt das 4. Buch (De motu) des Anti-Lucretius: vgl. insbesondere AL 4,933-1124 über die Gravitationstheorie.

14 Vgl. René Descartes: Die Prinzipien der Philosophie. Lateinisch-deutsch. Übersetzt und herausgegeben von Christian Wohlers. Hamburg 2005 (darin Teil 3: De mundo adspectabili).

15 Ein Beispiel von vielen: Ernest J. Ament: The Anti-Lucretius of Cardinal Polignac. In: TAPhA 101 (1970), S. 29-49: „We could smile, for instance, when he rejects his friend Newton's ,new theory' of the universal attraction of matter to explain weight [...] and his insistence on void to explain movement, did not this rejection evidence how slow to any age is the prevalence of truth over age-old error" (S. 40).

16 Vgl. dazu ausführlich Tanja Thern: Descartes im Licht der französischen Aufklärung. Studien zum Descartes-Bild Frankreichs im 18. Jahrhundert. Heidelberg 2003, S. 129-219. Thern geht auf Polignac allerdings nur en passant ein.

17 Einsteins Hypothese der Gravitationswellen wurde 2015 mit dem ,Laser Interferometer Gravitational Wave Observatory' (LIGO) experimentell nachgewiesen, wofür die Wissenschaftler 2017 den Nobelpreis für Physik erhielten. 
Kern war die Idee ja durchaus richtig, dass es keine instantane Fernwirkung ohne Übertragungsmedium geben kann. ${ }^{18}$

An einem konkreten Beispiel soll im Folgenden erläutert werden, wie Polignac einen bestimmten naturwissenschaftlichen Sachverhalt und seine Begründung poetisch dargestellt hat. Ausgewählt wurde die sogenannte Präzession der Erdachse, weil sich hieran die jeweiligen Ansätze in ihren unterschiedlichen Zugriffsmodi sehr schön demonstrieren lassen. Zur Erklärung muss (mit Polignac) ein wenig weiter ausgeholt werden.

Die Bewegung der Erde setzt sich nach den Erkenntnissen der zeitgenössischen Astronomie um 1700 aus drei Komponenten zusammen: Die erste Bewegung ist der jährliche Umlauf der Erde um die Sonne, der den Kepler'schen Gesetzen folgt; die zweite Bewegung ist die tägliche Drehung der Erde um ihre Achse, und die dritte ist eben die sogenannte Präzessionsbewegung, bei der die Erdachse wie bei einem Kreisel die Mantelfläche eines Kegels beschreibt. ${ }^{19}$ Ein vollständiger Umlauf dauert ca. 26.000 Jahre. $^{20}$ Die beiden ersten Bewegungen erfolgen (vom Himmelsnordpol aus gesehen) gegen den Uhrzeigersinn, die dritte Bewegung ist gegenläufig, d. h. im Uhrzeigersinn, so dass die Achse scheinbar ,vorgeht‘ (daher der Begriff praecessio). Dies schlägt sich in der Differenz zwischen dem sogenannten tropischen Jahr (Erdumlauf relativ zum Frühlingspunkt der Ekliptik) und dem siderischen Jahr (Erdumlauf relativ zur Position am Fixsternhimmel) nieder, die ca. 1/26.000 Jahr (= ca. 20 Minuten) beträgt: Das tropische Jahr ist um diesen Betrag kürzer, die astronomische Uhr geht gewissermaßen vor. Das Phänomen ist mindestens seit Hipparch (um 190-120 v.Chr.) bekannt, der aufgrund von Sternkartenvergleichen die Präzession entdeckte und auf ca. $1^{\circ}$ pro Jahrhundert abschätze (der tatsächliche Wert beträgt ca. $1,4^{\circ}$ pro Jahrhundert). ${ }^{21}$ Kopernikus erkannte, dass die Präzession auf die Kreiselbewegung der Erde zurückzuführen ist; ${ }^{22}$ die Ursache dieser Bewegung blieb freilich vorerst unbekannt.

18 Nach Einstein ist der (gekrümmte) Raum selbst das Medium, und die Gravitation breitet sich nicht instantan, sondern mit Lichtgeschwindigkeit aus.

19 Die periodischen Schwankungen der Präzessionslinie, die sogenannte Nutation, wurden erst 1728 entdeckt und waren Polignac zum Zeitpunkt der Abfassung des 8. Buches offenbar unbekannt. Daraus ergibt sich ein klares Argument für die Datierung bereits um 1700 wie für die anderen Bücher. Auch eine spätere Berücksichtigung der Nutation ist nicht erfolgt, was gegen eine Überarbeitung und Anpassung, etwa durch Rothelin, spricht.

20 Aufgrund verschiedener Faktoren, z. B. des Einflusses der Planetengravitation, schwankt der exakte Wert und liegt bei etwa 25.700 bis 25.800 Jahren.

21 Hipparchs Erkenntnisse sind im Almagest des Ptolemaios überliefert (Hg. von Johan Ludvig Heiberg. Leipzig 1898), dort Buch 3, Kap. 1, S. 191-192.

22 Nicolaus Copernicus: De revolutionibus orbium caelestium. Nürnberg 1543, lib. 3, cap. 1. 
Im späten siebzehnten und frühen achtzehnten Jahrhundert kam es, wie erwähnt, zu heftigen Kontroversen zwischen den Vertretern der Newton'schen Gravitationstheorie und der Cartesianischen Wirbeltheorie, die unterschiedliche Modelle für eine Erklärung der Himmelsbewegungen anboten. ${ }^{23}$ Die Ursache der Präzession liegt nach Newton (und nach der heutigen Physik) in der Tatsache, dass die Erde keine vollkommene Kugel, sondern ein an den Polen abgeplattetes Ellipsoid ist (der Poldurchmesser ist um ca. 1/300 kürzer als der Äquatordurchmesser). Die Gravitationskräfte von Sonne und Mond greifen am Äquatorwulst an und zwingen die Erde in eine Kreiselbewegung, die sich mithilfe der klassischen Physik theoretisch berechnen lässt. Die (annähernde) Übereinstimmung mit den Beobachtungsdaten war so beeindruckend, dass sie dem Newton'schen Modell schließlich zum Durchbruch verhalf, obwohl die Kernhypothese, d. h. die Fernwirkung der Gravitation durch den leeren Raum, rational unerklärbar blieb. Was die Cartesianer, und in ihrem Gefolge eben auch Polignac, an den Newtonianern kritisierten, war genau das, was einst Cicero gegen die Mantik ins Feld führte. Gegen die pragmatische Haltung der Mantiker, es komme darauf an, dass die Weissagung funktioniere, und es sei unerheblich, warum sie funktioniere (so etwa Ciceros Bruder Quintus in De divinatione), stellte Cicero die (sehr neuzeitlich klingende) Forderung, die Naturphänomene müssten prinzipiell rational erklärbar sein. ${ }^{24}$ Auch Polignac bezeichnet Newtons Gravitationstheorie als einen Rückfall in vorneuzeitliche Magie:

Fisa suis numeris Neutonia secta reciso impulsu magicis totum dedit artibus orbem.

(AL 4,933-934)

Im Vertrauen auf ihre mathematischen Formeln hat die Newton'sche Sekte die Impulstheorie aufgegeben und die ganze Welt magischen Künsten ausgeliefert.

Im Gegensatz zu Newton bot nach Polignac die Cartesianische Wirbeltheorie (hier Impulstheorie genannt) sehr wohl eine kausale Erklärung der Himmelsbewegungen an. Danach ist der Raum nicht leer (dem Streit um das Vakuum ist das gesamte 2. Buch De inani des Anti-Lucretius gewidmet), ${ }^{25}$ sondern vollständig mit einer sehr feinen Materie, dem sogenannten Äther, gefüllt, der sich in

23 Siehe oben Anm. 16.

24 Cic. Div., 1,15 (Quintus): „non quaero cur, quoniam, quid eveniat, intellego.“ Dagegen Div., 2,60 (Marcus): „causam igitur investigato in re nova atque admirabili, si poteris; si nullam reperies, illud tamen exploratum habeto, nihil fieri potuisse sine causa.“

25 Es ist nicht zufällig das erste der ,naturwissenschaftlichen` Bücher. Die Existenz des Leeren ist eine Kernhypothese der epikureischen und neuzeitlichen Physik, die man nach Polignac verachten muss ,wie Narrheiten und Traumgespinste einer verrückten Sekte“ (,delirantis uti lusus et somnia sectae“: AL 2,1164). Indem Polignac argumentiert, die Annahme eines Raums 
ständiger Bewegung befindet und die in ihm befindlichen Körper mit sich reißt. ${ }^{26}$ Die Kraft, die die Bewegung der einzelnen Körper verursacht, ist also keine unerklärliche Fernwirkung, sondern eine durch unmittelbare Berührung sich fortpflanzende Stoßkraft. In Bezug auf das Sonnensystem muss man sich das so vorstellen, dass die Planeten von unterschiedlich schnellen ,Wirbeln“, d. h. kreis- bzw. ellipsenförmigen Materieströmen mitgeführt werden. Die Geschwindigkeit der Wirbel nimmt dabei von innen nach außen ab, d. h. die inneren Planeten drehen sich schneller um die Sonne als die äußeren. In diesem noch relativ einfachen Modell muss aber nun auch die Bewegung der Erde um sich selbst sowie - als besondere Herausforderung - die Präzession erklärt werden. Dies erweist sich als nicht leicht, und deshalb stellt Polignac, nach dem Vorbild der antiken Lehrdichter, ${ }^{27}$ der entsprechenden Passage einen eigenen Musenanruf voran: ${ }^{28}$

Tu mihi nunc, praeclara novum Sapientia lumen, tu mihi coelestes animos infunde roganti, Uranie tu vera, tuas dum prosequor artes et succensus amore tui raptusque per auras ultima siderei motus arcana recludo. Duc igitur, qui te tua per vestigia quaerit, ne spatio immenso vagus aut incertus aberret.

(AL 8,768-774)

Du, strahlende Weisheit, flöße mir neue Erkenntnis, flöße mir deinen himmlischen Geist ein, ich bitte dich, du wahre Himmelsmuse (Urania), während ich deine Wissenschaft verfolge und, entflammt von Liebe zu dir und durch die Lüfte getragen, die letzten Geheimnisse der Bewegung der Himmelskörper erschließe. Führe also den, der dich auf deinen Spuren sucht, auf dass er nicht im unermesslichen Raum unstet und unsicher abirre.

Bemerkenswert ist die Betonung der Neuheit der Erkenntnis und der Bezug auf die „letzten Geheimnisse“, die es zu lüften gelte. Dass dies mehr als ein bloßer

ohne Inhalt sei sinnlos, weil ,Raum‘ kein Ding, sondern eine bloße Anschauungsform sei, nimmt er Kantische Gedanken vorweg; darüber hoffe ich andernorts genauer zu handeln.

26 Die Existenz des Äthers hatte Polignac bereits im 2. Buch behandelt (AL 2,650-777). Diese sogenannte Ätherhypothese hielt sich hartnäckig und konnte erst Ende des neunzehnten Jahrhunderts durch das Michelson-Morley-Experiment (Konstanz der Lichtgeschwindigkeit im leeren Raum, auch gegen den angeblichen Widerstand des Äthers) widerlegt werden, ein Meilenstein auf dem Weg zur speziellen Relativitätstheorie.

27 So z. B. in Vergils Georgica vor der Behandlung der Bugonie: Georg., 4,315.

28 Zitiert wird nach der Erstausgabe: Anti-Lucretius, sive de Deo et Natura libri novem. Eminentissimi S.R.E. Cardinalis Melchioris de Polignac opus posthumum; Illustrissimi Abbatis Caroli d'Orleans de Rothelin cura et studio editioni mandatum. Paris 1747. Orthographie und Interpunktion wurden leicht modernisiert. 
Topos ist, ergibt sich daraus, dass Polignacs Erklärung der Präzession nach der Cartesianischen Wirbeltheorie, soweit mir bekannt ist, die erste (und einzige) ihrer Art ist. ${ }^{29}$ Bevor Polignac freilich zu dieser ,dritten Bewegung‘ kommen kann, muss er zuvor die erste und zweite Bewegung erklären, wobei er teilweise auf frühere Passagen des achten Buches verweist.

Erste Bewegung:

Sunt duo materiae tamquam tabulata fluentis ducentisque globum. Superis quae partibus instat, uberior sane, quia maiorem occupat arcum, sed properat lente; minor est inferna tenentis copia, quandoquidem breviore includitur arcu, sed fuga vividior. Prius haec manifesta reliqui. Pensatis utrimque modis ac viribus, unum fit medium quoddam necnon aequabile momen, quo simul omne globi corpus pulsatur ab omni flumine: nam solidi partes ac membra cohaerent, etsi tanguntur varie, neque flectitur axis.

Motus hic assiduus (meministi) perficit annum.

(AL 8,775-786)

Es gibt gleichsam zwei Stockwerke fließender Materie, die die (Erd-)Kugel ${ }^{30}$ mit sich führen: Die Materie, die auf den oberen Teilen lastet, ist zwar massereicher, weil sie den größeren Bogen umspannt, fließt aber langsam; die Menge (der Materie), die das untere Stockwerk besetzt, ist geringer, da sie von einem kürzeren Bogen eingeschlossen wird, bewegt sich aber lebhafter voran. Das habe ich zuvor ja hinreichend erklärt (vgl. AL 8,559-683). Da die Eigenschaften und Kräfte auf beiden Seiten ausgewogen sind, ergibt sich ein bestimmter mittlerer, und zwar gleichmäßiger Impuls, durch welchen der gesamte Erdkörper vom Gesamtstrom angestoßen wird. Die Teile und Glieder eines festen Körpers hängen ja zusammen, auch wenn sie verschieden berührt werden, und die Achse wird dadurch nicht gebeugt. Diese dauerhafte Bewegung (du erinnerst dich) bewirkt den Jahresumlauf. ${ }^{31}$

Polignac führt hier aus, dass nach der Wirbeltheorie der äußere (d. h. sonnenabgewandte), massereichere Wirbel langsamer fließt als der innere (d. h. sonnenzugewandte), masseärmere; die Erde selbst wird dabei als ganze (da sie ein starrer Körper ist und nicht mehrere verschiedene Impulse aufweisen kann) mit einem mittleren, konstanten Impuls bewegt. Physikalisch lässt sich dies so

29 Das Problem war anscheinend zu speziell; auch Descartes selbst war im dritten Teil seiner Principia (1644), in dem er die Wirbeltheorie darlegt, nicht darauf eingegangen (s. oben Anm. 14).

30 Die Ausführungen gelten für alle Planeten; da im Folgenden aber immer speziell die Erde angesprochen ist, wird in der Übersetzung jeweils die Erde stellvertretend für die Planeten genannt, auch wenn die Formulierung bisweilen allgemeiner ist.

31 Gemeint ist also die Bewegung des gesamten Planetensystems gegen den Uhrzeigersinn. 
erklären, dass - bei unterschiedlichen Massen und Geschwindigkeiten - der Impuls der beiden Wirbel gleich ist: Es gilt $m_{1}>m_{2}$ und $v_{1}<v_{2}$, und zwar in der Weise, dass bei entsprechenden Werten Impulsgleichheit vorliegt: $m_{1} \cdot v_{1}=m_{2} \cdot v_{2}$.

Dass die Bahngeschwindigkeit der Erde aufgrund der Elliptizität der Bahn tatsächlich leicht variiert, bleibt hier unberücksichtigt, weil es, wie im unmittelbar vorhergehenden Abschnitt (AL 8,684-767) erläutert, die Darstellung an dieser Stelle unnötig verkomplizieren würde; es kommt Polignac jetzt vielmehr auf die Erklärung der zweiten und dritten Bewegung an. Diese resultieren letztlich aus der erwähnten Differenz der oberen und unteren Wirbelgeschwindigkeit.

Zweite Bewegung:

Causa vices eadem noctis parit atque diei.

Nam cum terra natet superis velocior undis, segnior infernis, partes lambentibus imas obicit illa moram et postica parte resistit. Ac veluti magnis ubi molibus agger aquarum intercludit iter, gemit amnis et agmine fluctus agglomerant sese cumulatim, culmina donec ardua transiliunt et largo flumine inundant: incurrit sic materies effusa natantem in scopulum, quatiens a tergo quidquid in altum prominet. At quoniam densum pervadere corpus haud potis est neque segnitiem accelerare morantis, tantum reprimitur, quanto ipsa citatior illo est. Non habet ut refluat, cum posteriore prematur; nec subterfugio locus est, inhibetur ab illo flumine, quod semper velocius alluit infra; quin et centrifugae vires a sole repellunt: cogitur in superas ergo conscendere partes, lenior est ubi materiae progressus euntis, quam concedentem facili conamine vincit. Sic valido appulsu molem amplexata rotundam desuper adradens ferit inclinatque cacumen. Huius descensu liquor interceptus ad ima truditur impellitque globum subtusque movendo erigit infernas partes. Ea causa vicissim dimidio dat dimidium succedere semper.

(AL 8,787-812)

Dieselbe Ursache bewirkt auch den Wechsel von Tag und Nacht. Denn da die Erde schneller als die oberen und langsamer als die unteren Wogen schwimmt, setzt sie letzteren, d. h. den die unteren Teile berührenden Wogen, eine Verzögerung entgegen und bremst mit ihrem hinteren Teil. Und wie ein Fluss, wo ein Deich mit großer Masse den Weg des Wassers versperrt, heftig rauscht und sich die Fluten Zug um Zug immer mehr anstauen, bis sie die Deichkrone überspringen und sich in breitem Strom (ins Land) ergießen: So 
prallt die strömende Materie gegen den schwimmenden Felsen (= die Erde) und schlägt von hinten gegen alles, was in die Höhe ragt. Da es jedoch nicht möglich ist, den dichten Körper zu durchdringen oder die träge Masse zu beschleunigen, wird sie um so viel zurückgedrängt, um wie viel schneller die Materie als jener Körper ist. Die Erde kann nicht rückwärts fließen, da sie von der hinteren Seite gedrückt wird; sie kann auch nicht nach unten ausweichen, da sie von jenem Strom, der ständig unterhalb fließt und schneller ist, gehindert wird; außerdem treibt die Zentrifugalkraft sie von der Sonne weg: Sie ist also gezwungen, zu den oberen Teilen hin aufzusteigen, wo der Vortrieb der fließenden Materie geringer ist; diese kann sie mit leichter Anstrengung verdrängen, da die Materie zurückweicht. Auf diese Weise trifft (aber) die Materie, die die runde Erdmasse umfasst, diese mit kräftigem Druck von oben, schleift sie mit und beugt den obersten Teil (nach unten). Durch dessen Abstieg wird der Materiefluss abgefangen und nach unten gedrückt, treibt die Kugel weiter an und richtet die unteren Teile durch die Abwärtsbewegung auf. Diese Ursache bewirkt, dass die eine Hälfte der anderen in stetem Wechsel folgt.

Die tägliche Drehung der Erde um ihre Achse wird also dadurch verursacht, dass sie dem unteren, schnelleren Wirbel einen Widerstand entgegensetzt, was durch ein (nur teilweise passendes) Deich-Gleichnis veranschaulicht wird: Die Fluten stauen sich von hinten auf und überspülen schließlich den Deich, während die Erde jedoch dadurch in eine zusätzliche Drehbewegung versetzt wird. Sie kann ihre Bahn nicht verlassen; daher weicht diejenige Seite der Erde, die dem stärkeren Fließdruck unterliegt, nach ,oben', also in Richtung des langsameren Wirbels, aus, von dem sie wiederum erfasst und nach unten gedrückt wird. Ein sachliches Problem der obigen Beschreibung liegt darin, dass nicht klar gesagt wird, in welche Richtung die Drehung vonstattengeht: Ging Polignac davon aus, dass es nicht eigens erwähnt werden musste, da natürlich bekannt war, dass die Erddrehung von West nach Ost, d. h. ebenfalls gegen den Uhrzeigersinn, erfolgt? Oder ließ er die Darstellung bewusst vage, um eine Schwachstelle in der Argumentation zu kaschieren? Jedenfalls liegt hierin eine gewisse Unklarheit auf der phänomenologischen Seite. Darüber hinaus fragt sich allerdings auch, ob die Erklärung überhaupt sachlich korrekt ist: Müsste sich nicht, intuitiv betrachtet, die Kugel in die andere Richtung drehen, weil der untere Wirbel schneller ist? Das Problem sah offenbar Polignac selbst, denn er fügt zur weiteren Erklärung noch folgenden Passus hinzu, in dem er bereits die dritte Bewegung andeutet:

Vincitur idcirco superi ubertate fluenti inferius, quamquam citiori vincere motu posse videbatur totamque invertere terram semper ad occasum, sinerent obstantia. Verum non ita concedit, quin vires explicet omnes. Et quia ter novies millena parte (parum deest) ocius incurrit, momen telluris in ortum ter novies etiam millena parte retardat. 
Inde fit, ut rediens expleto denique terra

curriculo stellas iam non offendat eodem

in puncto coeli, nec eo se dirigat axis,

quo directus erat, quando prior exiit annus:

Ast ignara rei stellas tantisper ab ortu

descivisse putat, quamvis desciverit ipsa.

(AL 8,813-826)

Durch die größere Masse der oberen Strömung wird also die untere besiegt, obwohl es schien, als könne diese durch ihre schnellere Bewegung jene übertreffen und die ganze Erde ständig nach Westen drehen, ließe deren Widerstand es zu. Tatsächlich aber erlaubt (jene obere) es nicht, dass (die untere) all ihre Kräfte entfaltet. Und weil diese (d.h. die untere Strömung) um den 27.000sten Teil - es fehlt nur wenig daran - schneller läuft, verzögert sie die Antriebskraft der Erde nach Osten ebenfalls um den 27.000sten Teil. Daher geschieht es, dass die Erde, wenn sie ihren Umlauf schließlich beendet hat, bei ihrer Rückkehr die Sterne nicht mehr an demselben Punkt des Himmels antrifft und sich die Achse nicht mehr dahin ausrichtet, wohin sie gerichtet war, als das vorherige Jahr endete: Vielmehr glaubt sie, in Unkenntnis der Sachlage, dass die Sterne ein klein wenig von Osten abgewichen seien, obwohl (tatsächlich) sie selbst abgewichen ist.

Polignac räumt also ein, dass die Strömungsmechanik der Wirbel eigentlich eine Drehung der Erde nach Westen (im Uhrzeigersinn) verursachen müsste; allein der Widerstand der Erde lasse dies nicht zu (sinerent obstantia: der Plural deutet eine Summe vieler einzelner Widerstände an): Dieser entstehe durch die Unregelmäßigkeit der Erdoberfläche (quidquid in altum prominet: V. 796-797), bei der sich die größere Masse des oberen Wirbels stärker auswirke. Physikalisch kann dies nicht mehr plausibel gemacht werden; es ist der Versuch, unter Beibehaltung der Wirbeltheorie die Phänomene zu retten. Indem er aus der Not eine Tugend macht, will Polignac allerdings nun die höhere Geschwindigkeit des unteren Wirbels zur Erklärung der ,dritten Bewegung' nutzen: Danach bremst der untere Wirbel die Drehung der Erdachse um 1/27.000 pro Jahr ab (nach Polignac ein Näherungswert: tatsächlich ca. 1/26.000, siehe unten) und verursacht so das Phänomen der Präzession, das diesmal sehr anschaulich durch eine personifizierte Erde, die sich über die veränderte Position der Fixsterne wundert, beschrieben wird. Weitere Erklärungen folgen:

Dritte Bewegung:

Tertius hic motus geminis contrarius esse debuit, ut constans terrae positura maneret in fluido, vi cuius eunt annusque diesque. Nempe aliud cum sit centrum gravitatis in orbe terreno molisque aliud, pars altera terrae quae gravior, pars quae levior, non sicut oportet motum dividerent, ex uno fine minorem, 
maiorem ex alio conum describeret axis.

Ergo, quod nimium superest, mora facta recidit, temperat exaequans motus et corrigit axem.

Is cum aequatorem, quo ecliptica linea distat, ut dictum est, gradibus prope quatuor et viginti ad perpendiculum scindat; sic distat ab illa sexaginta et sex gradibus prope: qui status axi perpetuus, quocumque modo se terra revolvat, sive diem faciens sive annum aut saecla ducenta cum sexaginta; quibus actis terra revertens huc unde exierat primum, tunc reddita priscis astra locis credet, cernens ea rursus ibidem.

(AL 8,827-845)

Diese dritte Bewegung musste zu den beiden anderen gegenläufig sein, damit die Position der Erde im Fließmedium, das Jahres- und Tageslauf mit seiner Kraft bestimmt, konstant blieb. Obwohl nämlich das Zentrum der Schwere in der Erdumlaufbahn (= die Sonne) und das der Erdmasse selbst verschieden sind, heißt das nicht, dass der (sonnennähere) Teil der Erde, der schwerer ist, und der (sonnenfernere), der leichter ist, deshalb verschiedene Bewegungen aufweisen und dass die Achse am einen Ende einen kleineren, am anderen einen größeren Kegel beschreiben würde. Folglich beseitigt die eingetretene Verzögerung das, was überschüssig ist, gleicht die Bewegung mäßigend aus und korrigiert die Achse. $\mathrm{Da}$ diese den Äquator, von dem die Ekliptik, wie dargelegt wurde, um etwa 24 Grad abweicht, senkrecht schneidet, ist die Achse von der Ekliptik etwa 66 Grad entfernt: Dieser Abstand bleibt für die Achse konstant, wie auch immer sich die Erde bewegt - sei es dass sie einen Tages-, einen Jahres- oder einen Lauf von 260 Jahrhunderten zurücklegt. Wenn die Erde nach diesem Zeitraum wieder dahin zurückkehrt, von wo sie anfangs ausgegangen war, wird sie glauben, die Sterne stünden wieder an der alten Stelle, da sie dieselben ebendort wiedersieht.

Das Phänomen der Präzession als solches ist vollkommen korrekt dargestellt, sieht man von etwas unpräzisen Zahlenwerten ab (Neigung der Erdachse gegen die Ekliptik um $66^{\circ}$ statt exakt $66,5^{\circ}$; Präzessionszyklus einmal mit 26.000 Jahren angegeben, einmal mit 27.000 Jahren). Die Erklärung erfolgt aber nun völlig überraschend - nicht mithilfe der Wirbeltheorie, sondern mithilfe der Schwerkraft, die auf die sonnennäheren und -ferneren Teile des Erdkörpers unterschiedlich einwirke! Das kommt der tatsächlichen Ursache der Präzession, nämlich der ,Gezeitenwirkung' von Sonne und Mond auf den Äquatorwulst, schon sehr nahe. Gewiss, im Lehrgedicht können traditionell verschiedene Erklärungsansätze durchaus nebeneinander stehen, aber es ist schon merkwürdig, dass Polignac hier anscheinend ohne Bedenken das Newton'sche Modell, das er sonst ablehnt, zur Erklärung heranzieht. Möglicherweise liegt hier aber auch eine Passage vor, die mit dem Vorherigen nicht harmonisiert wurde (auffällig ist auch die abweichende genauere Zeitangabe von 26.000 gegenüber 
27.000 Jahren): ${ }^{32}$ Dem Lehrgedicht fehlt ja die letzte Redaktion durch den Autor, und der Herausgeber fügte häufig nur ,fliegende Blätter` zusammen. ${ }^{33}$ Auch der Anschluss der folgenden Passage ist verdächtig:
Iamque vides uno quam belle ac simplice motu
magna planetarum magno volvantur in orbe
corpora perpetuoque in se conversa rotentur.
$\mathrm{Nec}$ te is detineat nodus (quem solvere primo
conatu promptum est, Newtoni industria quamvis
nexuerit) fluidam crassis obsistere molem
corporibus, motum hinc minui tandemque futurum,
ut pereat. Verum hoc esset, si torpida moles
stagnaret fluidi aut adverso concita motu
obluctaretur contra venientibus astris:
At nemo alterutrum dicat. Fluit incitus aether, quo sphaerae currunt; simul uno more feruntur una vi solis pulsuque moventur eodem.
Sic non est, aether sphaeris ut mole resistat.
Aiunt praeterea transversos ire cometas
aethera per medium neque concordare planetis.

(AL 8,846-861)

Schon erkennst du jetzt, wie schön und mit wie einziger und einfacher Bewegung die großen Körper der Planeten sich auf ihrer großen Bahn bewegen und sich (dazu) ständig um sich selbst drehen. Es soll dich aber nicht der ,Knoten' abhalten (der auf einen Schlag zu lösen ist, obwohl der scharfsinnige Newton ihn geknüpft hat), dass das Fließmedium kompakten Körpern Widerstand leiste, die Bewegung sich dadurch verlangsame und schließlich zum Stillstand kommen werde. Dies träfe zu, wenn die Masse des Fließmediums starr wäre und stillstünde oder, durch eine Gegenbewegung angetrieben, den entgegenkommenden Himmelskörpern gewaltsam Widerstand leistete: Doch niemand behauptet das, weder das eine noch das andere. Der Äther fließt (seinerseits), in die Richtung angetrieben, in die auch die Kugeln rollen; auf ein und dieselbe Weise werden auch sie gleichzeitig dahingetragen, zusammen mit der Kraft der Sonne, und von demselben Impuls fortbewegt. So ist es nicht der Fall, dass der Äther den Kugeln aufgrund seiner Masse widersteht. (Außerdem argumentieren sie, dass die Kometen ja schräg mitten durch den Äther gingen und nicht mit den Planeten übereinstimmten.) $)^{34}$

32 Liegt die Abweichung in der poetischen Ausdrucksweise ter novies millena parte gegenüber saecla ducenta cum sexaginta begründet? Aber cum septuaginta hätte mit einigem guten Willen auch ins Metrum gepasst.

33 Dazu äußert sich der ,Endherausgeber‘ Charles LeBeau, der nach dem Tod des Abbé de Rothelin das doppelt verwaiste Werk zur Publikation brachte, ausführlich im Vorwort. Zur Publikationsgeschichte siehe die Angaben oben in Anm. 10.

34 Die letzten beiden Verse beziehen sich auf einen Einwand der Äther-Gegner und bilden den Übergang zum nächsten Thema, den Kometen (AL 8,860-898). 
Der Newton'sche Einwand gegen den Äther wird hier mit grundsätzlichen Überlegungen zur Dynamik des Fließmediums entkräftet, die freilich an dieser Stelle fehl am Platz sind: Dass die Kugeln quasi mit dem Strom schwimmen, wie hier gesagt wird, ist zwar im Groben richtig, aber zuvor war ja gerade damit argumentiert worden, dass die Geschwindigkeiten der Wirbel unterschiedlich und diese auf die eine oder andere Weise Widerständen ausgesetzt seien. Zudem ging es in dem besprochenen Abschnitt um drei unterschiedliche Bewegungen der Erde, während hier von der ,einen, einfachen Bewegung der Planeten' (unus simplex motus) gesprochen wird. Das legt den Schluss nahe, dass dieser Passus bei der Redaktion an die falsche Stelle geraten ist. ${ }^{35}$

Fassen wir zusammen. Die drei Bewegungen des Planeten Erde (Umlauf um die Sonne, Drehung um die Achse und Präzession) werden astronomisch korrekt und einigermaßen präzise beschrieben. Die Erklärungen mithilfe der Cartesianischen Wirbeltheorie sind aus heutiger Sicht naturgemäß unzureichend und widersprechen zum Teil physikalischen Tatsachen (etwa der Strömungslehre), wie sie heute bekannt sind. Man muss Polignac aber zugutehalten, dass er die Probleme nicht verschweigt, sondern offen benennt, auch wenn er dadurch den Leser in gewisser Weise unbefriedigt zurücklässt und nicht restlos überzeugen kann. Denn trotz der sachlichen Argumentationsschwierigkeiten verzichtet Polignac darauf, rhetorische Mittel im Übermaß einzusetzen: In der gesamten besprochenen Passage (von immerhin fast 100 Versen) finden wir z. B. nur ein einziges Gleichnis, das über etwas Lokalkolorit hinaus (Deiche hatte Polignac während seines Aufenthaltes in den Niederlanden gesehen) wenig vermittelt, und eine poetisch ansprechende Personifikation der Erde, die sich aber auf einen ganz unstrittigen Sachverhalt (die scheinbar veränderte Position der Fixsterne) bezieht. Anders als im 2. und 4. Buch fehlt jetzt auch jegliche Polemik gegen die „Sekte“ Newtons. Der vorangestellte Musenanruf bekräftigt vielmehr die Schwierigkeit des Gegenstands und die Gefahr, sich im unermesslichen Raum zu verirren. Dass die angebotenen Erklärungen heute überholt sind, ist daher nicht entscheidend (auch Newton ist ja längst überholt); entscheidend ist die Unbeirrbarkeit, mit der Polignac eine rationale Erklärung der Naturphänomene verficht. Und das wiederum ist gar nicht anti-lukrezisch. ${ }^{36}$

35 Möglicherweise gehört die Passage eher ins 2. Buch (angeblicher Widerstand des Äthers als Argument für die Vakuum-Hypothese) oder ins 4. Buch (angebliche Bremswirkung des Äthers), das im Übrigen einen regelrechten Hymnus an den Äther enthält (AL 4,541-574).

36 Für Beratung in physikalischen Fragen danke ich meiner Tochter Felicia Glei. 